\title{
Ambiente de luz del sotobosque de una selva nublada andina: estructura del dosel y estacionalidad climática
}

\author{
Ana Quevedo ${ }^{1}$, Teresa Schwarzkopf ${ }^{2}$, Carlos García $^{2} \&$ Mauricio Jerez ${ }^{3}$
}

1. Facultad de Ciencias Forestales y Ambientales. Escuela Técnica Superior Forestal (ETSUFOR), Universidad de Los Andes; anamer2@gmail.com

2. Facultad de Ciencias. Instituto de Ciencias Ambientales y Ecológicas (ICAE), Universidad de Los Andes; tschwarzkopf@gmail.com, carlosgarnu@gmail.com

3. Facultad de Ciencias Forestales y Ambientales. Centro de Estudios Forestales y Ambientales de Postgrado (CEFAP), Universidad de Los Andes; jerezorama@gmail.com

$$
\text { Recibido 17-XI-2015. Corregido 15-IV-2016. Aceptado 16-V-2016. }
$$

\begin{abstract}
Light environment in the understory of an Andean cloud forest: canopy structure and climatic seasonality. The light environment in the understory of cloud forests is highly heterogeneous and determined by species composition, canopy structure, site conditions, and seasonality. This study was carried out at San Eusebio cloud forest, Venezuela (2 300 - 2500 masl). The impact of canopy structure variations on understory light availability was estimated in the dry (December-February) and rainy (March-November) seasons, in sites under continuous canopy cover and gaps of various sizes. Hemispherical photographs were taken to estimate the percentage of canopy openness, leaf area index, percentages of transmitted direct and diffuse light, and duration and frequency of sunflecks. A light index was calculated from the relative proportions of direct and diffuse light transmitted to the understory. For most variables, there were significant differences between seasons, as well as among different gap sizes and under closed canopy. The light index was low $(0.25$ to 26 of a maximum $=$ 100 ), even for the largest gaps, indicating a highly shaded light environment, especially beneath closed canopy in the rainy season. Patterns and interactions among factors were analyzed (gaps vs. continuous canopy, gap sizes, location within the gaps, and seasonality) with a mixed effects repeated measures Anova design. Results showed that the amount of light reaching the understory is low in both gaps and closed canopy. However, small but significant differences in light availability existed for both seasonality and magnitude of the perturbations. These differences could contribute to explain the dynamics of tree species regeneration in this forest. The knowledge of the factors conditioning light availability in the understory where tree regeneration begins is crucial in cloud forests because of energetic limitations in this ecosystem, and might be essential for future restoration and conservation plans concerning the preservation of the diversity and integrity of these forests. Rev. Biol. Trop. 64 (4): 1699-1707. Epub 2016 December 01.
\end{abstract}

Key words: gaps, hemispherical photographs, light index, sunflecks, direct and diffuse light.

El ambiente lumínico en el sotobosque de selvas tropicales es determinante en la supervivencia y crecimiento de las especies arbóreas en sus primeras fases de desarrollo. Este ambiente es muy heterogéneo debido a las complejas interacciones entre la luz incidente y la estructura de la vegetación que varían de acuerdo a factores como el clima, la topografía y la dinámica del bosque. La estructura del dosel, no es estática, sino que cambia continuamente debido a perturbaciones que resultan de la interacción entre factores ambientales y la vegetación. Como consecuencia, el ambiente de luz en el bosque es espacial y temporalmente heterogéneo (Endler, 1993; Pearcy, 2007). La formación de claros en el dosel del bosque ha sido considerada por mucho tiempo como el proceso que dirige el comienzo de la regeneración de los bosques tropicales. La perturbación local que crea un claro altera las variables 
microclimáticas, y por consiguiente, altera las condiciones de germinación y crecimiento. Específicamente, los claros y las variaciones estacionales del dosel pueden alterar considerablemente la cantidad de luz que llega al sotobosque y determinar el establecimiento y desarrollo de la regeneración. Este aspecto es fundamental para el éxito de planes de conservación y restauración de ecosistemas boscosos.

En las selvas nubladas (SN), la disponibilidad espacio-temporal de la luz en el sotobosque es baja debido a topografías montañosas, altas precipitaciones y la nubosidad persistente (Luna, Velázquez, \& Velázquez, 2001; Hogan \& Machado, 2002); a esto se suma la presencia de doseles relativamente densos (Schwarzkopf, Riha, Fahey, \& DeGloria, 2011). La regeneración exitosa de las especies arbóreas implica la existencia de mecanismos adaptativos a las limitaciones energéticas impuestas por la baja incidencia de radiación solar que caracteriza estos ecosistemas (Acevedo, Ataroff, Monteleone, \& Estrada, 2003), lo cual a su vez determinaría la estructura y diversidad del bosque. La supervivencia de los árboles en su fase juvenil depende en gran medida de su capacidad para aclimatarse o aprovechar cambios en la disponibilidad de luz directa y difusa en el sotobosque determinada por la estructura del dosel. Los rayos de sol que llegan al sotobosque dependen de atributos tales como: la altura y flexibilidad del dosel, el tamaño de las hojas, el viento y la nubosidad (Pearcy, 2007), los cuales son muy variables en duración y magnitud (Cescatti \& Niinemets, 2004; Pearcy, 2007). El impacto de las limitaciones energéticas en la regeneración de los árboles parece indudable, por tanto, nos propusimos explorar, mediante fotografías hemisféricas $(\mathrm{FH})$, las magnitudes y diferencias en el ambiente de luz en el sotobosque debidas a la estructura del dosel y entre las estaciones seca y lluviosa en la SN San Eusebio de Los Andes Venezolanos. Concretamente, nos preguntamos: ¿Cuán significativo es el aumento de la disponibilidad de luz en el sotobosque como resultado de la apertura de un claro? ¿Existen diferencias según el tamaño y edad del claro, así como en la zona (borde/centro) dentro del mismo? y ¿Es la estacionalidad en las precipitaciones un factor significativo de alteración del ambiente de luz en el sotobosque?

\section{MATERIALES Y MÉTODOS}

Área de estudio: El estudio se realizó en el Bosque Universitario San Eusebio, Mérida, Venezuela, localizado en la Cordillera de los Andes entre 2200 y 2500 msnm (8 $37^{\circ} 00^{\prime \prime} \mathrm{N}$ - 71'21'00" W). La precipitación oscila entre 1400 y $1560 \mathrm{~mm}$ y presenta un régimen biestacional, con estaciones seca (ES) (diciembre a febrero) y lluviosa (ELL) (marzo a noviembre). La temperatura media anual es de $14.9^{\circ} \mathrm{C}$ y el flujo fotosintético de fotones a campo abierto es de $1561 \pm 77.2 \mu \mathrm{mol} / \mathrm{m}^{2}$.s (García, Azócar, \& Rada, 1995). El relieve típico es de colinas redondeadas, con pendientes variables desde muy suaves a fuertes y topografía muy irregular (Márquez, 1990). El ecosistema se clasifica como bosque nublado montano. Es de carácter mixto, irregular, densamente cerrado, muy rico en especies con árboles colonizados por numerosas epífitas. Las familias arbóreas predominantes son: Lauraceae, Melastomataceae, Guttiferae, Euphorbiaceae, Myrtaceae y Podocarpaceae. Existen varias comunidades vegetales, entre ellas el bosque alto denso (BAD) con estratificación compleja y dosel a $30 \mathrm{~m}$ y los bosques bajos ralos con dosel no mayor a $15 \mathrm{~m}$ de altura. El estudio se realizó en el BAD, que posee tres estratos bien diferenciados: el superior entre los 25 y $30 \mathrm{~m}$ de altura, con árboles emergentes de hasta $40 \mathrm{~m}$ de altura; el estrato intermedio entre 20 y $25 \mathrm{~m}$, y el inferior entre 10 y 15 m. El sotobosque está compuesto por regeneración arbórea, arbustos, lianas, palmas y herbáceas. Son frecuentes los helechos arborescentes del género Cyathea y bambúes del género Chusquea que puede formar espesos matorrales (Ramos \& Plonczak, 2007; Schwarzkopf et al., 2011).

Muestreo de campo: Para estudiar el efecto de la estructura del dosel, ésta se caracterizó en una serie de puntos según su grado 
de perturbación, desde zonas con dosel continuo o bosque no perturbado (BNP) hasta claros de diferentes tamaños y antigüedad, así como zonas dentro de claros. Para ello se estableció un muestreo sistemático sobre 32 ha, trazando alineaciones separadas cada $100 \mathrm{~m}$. El primer punto en cada alineación fue elegido aleatoriamente, y los sucesivos fueron ubicados a intervalos de $30 \mathrm{~m}$. Un punto dado se consideró claro, cuando: a) había interrupción abrupta del estrato superior del dosel; b) presencia de uno o varios árboles caídos; o c) cobertura del dosel $<90 \%$ determinada con un densiómetro de Lemmon. Los claros se delimitaron siguiendo el método de Brokaw (1985), luego se determinó su superficie y se clasificaron por tamaño como: "pequeño" (50-130 m²), "mediano" (131-170 m²) y "grande" (171-400 $\left.\mathrm{m}^{2}\right)$ de acuerdo a la variabilidad observada (49 a 446 $\mathrm{m}^{2}$ ). De 96 puntos evaluados, se eligieron al azar 15 sitios en BNP y 15 sitios en claros. Para cada claro se calificó como zona central un área equivalente al $30 \%$ de su superficie total y el $70 \%$ restante se consideró como borde o área de penumbra. Con la ayuda de baqueanos se estimó la antigüedad del claro, considerándose "recientes" aquellos con menos de cinco años de haber ocurrido y "antiguos" todos los demás (Apéndice digital). Para tomar las FH, en cada zona del claro se hizo una selección aleatoria de puntos, restringida a una distancia mínima de dos metros entre éstos, hasta abarcar la superficie total del claro. En los sitios de BNP se marcaron ocho puntos dentro de un círculo de radio $8.92 \mathrm{~m}$, situados a 3 y $6 \mathrm{~m}$ del centro en cada uno de cuatro cuadrantes. En cada punto $(\mathrm{n}=288,120$ puntos en BNP y 168 en claros) se tomaron dos FH (una en cada estación) para un total de 576 fotografías.

Ambiente de luz en el sotobosque: En cada punto se tomaron $\mathrm{FH}$ tanto en la ES (febrero) como en la ELL (octubre 2010) con una cámara digital Nikon COOLPIX P5000 de 10 megapíxeles provista de una lente hemisférica "Fisheye Converter" Nikon FC-E8. Este equipo se montó en un trípode, asegurándose la horizontalidad y posición del lente a $1.5 \mathrm{~m}$ de altura. Las fotografías se tomaron siguiendo el protocolo de Zhang, Jing y Miller (2005), basado en la determinación correcta del grado de exposición a la luz (control de la apertura del lente y velocidad de obturación). Mediante calibración previa en campo, se establecieron valores óptimos de apertura del lente de f5.3 a f5.4 y velocidades de obturación de $1 / 125$ a $1 / 250$, con ISO-200.

El procesamiento de las fotografías consistió en: a) diferenciar correctamente los pixeles que representan cielo abierto o vegetación mediante SIDELOOK (Nobis \& Hunziker, 2005). b) obtener las estimaciones de variables de estructura del dosel y de luz mediante el software Gap Light Analyzer (GLA) v. 2.0 (Frazer, Canham, \& Lertzman, 1999), que simula el régimen diario de radiación solar y la estructura de la vegetación para periodos considerados (diciembre-febrero y marzonoviembre). El GLA requiere ingresar datos de latitud, longitud, estación de crecimiento y su respectivo índice de nubosidad, altitud y pendiente del sitio, así como el umbral óptimo obtenido para cada foto para estimar las variables de luz y dosel. Para ello calcula la posición del sol durante cada periodo y la cantidad de luz teórica incidente por unidad de superficie (Frazer et al., 1999). Se estimó a) índice de área foliar (IAF); b) porcentaje de dosel abierto ( $\%$ DA); c) porcentaje de luz directa transmitida (\% TDIR): proporción de luz directa transmitida través del dosel con respecto a la radiación directa recibida por encima del dosel; d) porcentaje de luz difusa transmitida (\% TDIF): proporción de luz difusa transmitida través del dosel con respecto a la radiación difusa recibida por encima del dosel; e) duración promedio de los rayos de sol (DRS): minutos de luz directa que potencialmente podrían llegar a un punto en el sotobosque; y f) frecuencia de rayos de sol (FRS): número de rayos de sol de una duración específica (minutos) que potencialmente podría experimentar un punto en el sotobosque (Frazer et al., 1999). Además, se determinó el Índice de Luz (ILuz), el cual varía entre 0 (ausencia de luz) y 100 (cielo abierto), definido por Canham (1988) como: 
Iluz $=(($ Tdifusa $)($ Pdifusa $)+($ Tdirecta $)($ Pdirec ta)) (100), en donde Pdifusa y Pdirecta son las proporciones de radiación total difusa y directa recibidas por encima del dosel; y Tdifusa y Tdirecta son las proporciones de radiación directa y difusa transmitidas al sotobosque.

Se analizó si existían diferencias en las variables entre BNP y claros entre ES y ELL; además entre tamaños de claro $v s$. antigüedad, y tamaño vs. posición (centro-borde). Se realizó un análisis de varianza de efectos mixtos que tomó en cuenta la correlación entre medidas repetidas (las FH de cada estación se tomaron en los mismos puntos), desbalance en los tamaños de muestra, y la heterogeneidad de varianzas en los tratamientos. Se consideraron las posibles interacciones entre los factores. Los análisis se efectuaron mediante el procedimiento MIXED de SAS v 9.1 (SAS, 2004). Se estimaron las medias marginales (least squares means) para cada variable. Una prueba de Tukey-Kramer permitió hacer comparaciones entre medias para un factor considerando niveles fijos de otro (ej. diferencias en la variable ILuz entre estaciones para cada nivel del factor tipo de bosque: BNP-claros).

\section{RESULTADOS}

Estructura del dosel y variación estacional: Los resultados del ANOVA no mostraron una interacción significativa entre estación y perturbación de la estructura del dosel $(\mathrm{p}>0.15$ para todas las variables). Al comparar entre claros y BNP, el IAF fue significativamente mayor para el BNP independientemente de la estación ( $p$-valor $\leq 0.001$ ). Asimismo, al comparar la variación estacional dentro del BNP y dentro de los claros, el IAF fue significativamente mayor $(\mathrm{p}<0.05$, letras a,b) durante la ELL (Cuadro 1). Todas las demás variables fueron significativamente mayores para los claros $(\mathrm{P}$-valor $\leq 0.004)$,

CUADRO 1

Estimados de las variables de luz y estructura del dosel para BNP y claros $(\mathrm{n}=288, \mathrm{BNP}=120$, claros $=168)$ para cada estación

TABLE 1

Estimates of the light and canopy structure variables for BNP and gaps $(\mathrm{n}=288, \mathrm{BNP}=120$, gaps $=168)$ in each season. In parenthesis standard errors

\begin{tabular}{lllcc}
\multicolumn{1}{r}{ Variables } & \multicolumn{1}{c}{ Periodo } & BNP & Claros & P-valor \\
IAF & Seca & $5.2(0.1)^{\mathrm{b}}$ & $4.0(0.1)^{\mathrm{b}}$ & $<0.01$ \\
& Lluviosa & $5.6(0.1)^{\mathrm{a}}$ & $4.4(0.1)^{\mathrm{a}}$ & 0.01 \\
\% DA & Seca & $2.3(0.1)^{\mathrm{a}}$ & $4.9(0.2)^{\mathrm{a}}$ & $<0.01$ \\
& Lluviosa & $1.5(0.1)^{\mathrm{b}}$ & $4.1(0.2)^{\mathrm{b}}$ & $<0.01$ \\
\% TDIR & Seca & $4.0(0.2)^{\mathrm{a}}$ & $9.1(0.5)^{\mathrm{a}}$ & 0.04 \\
& Lluviosa & $3.1(0.2)^{\mathrm{a}}$ & $6.5(0.4)^{\mathrm{b}}$ & $<0.01$ \\
\multirow{2}{*}{ DRIF (min) } & Seca & $3.2(0.1)^{\mathrm{a}}$ & $8.0(0.4)^{\mathrm{a}}$ & $<0.01$ \\
& Lluviosa & $2.5(0.2)^{\mathrm{a}}$ & $6.6(3.3)^{\mathrm{b}}$ & $<0.01$ \\
FRS & Seca & $15.7(1.0)^{\mathrm{a}}$ & $34.6(2.9)^{\mathrm{a}}$ & $<0.01$ \\
& Lluviosa & $13.9(0.9)^{\mathrm{a}}$ & $34.1(2.6)^{\mathrm{a}}$ & $<0.01$ \\
& Seca & $12.3(0.7)^{\mathrm{a}}$ & $21.5(1.2)^{\mathrm{a}}$ & $<0.01$ \\
\hline
\end{tabular}

IAF: índice de área foliar; \% DA: porcentaje de dosel abierto; \% TDIR: porcentaje transmitido de luz directa; \% TDIF: porcentaje de luz difusa; DRS: duración promedio en minutos de los rayos de sol; FRS: frecuencia o número de rayos de sol de una duración específica.

En paréntesis errores estándar. Letras diferentes en las celdas indican diferencias estadísticamente significativas entre estaciones $(\mathrm{P}<0.05)$. El P-valor corresponde al nivel de significación de la comparación entre BNP y claros.

Different letters in the cells indicate statistically significant differences between seasons $(\mathrm{P}<0.05)$. The $\mathrm{P}$-value corresponds to the signification level for the comparisons between BNP and gaps. 
independientemente de la estación, excepto la FRS en la ELL (P-valor $=0.121)$.

Al comparar la variación estacional dentro de BNP y claros, las diferencias son menos importantes tanto en magnitud como en significación (Cuadro 1). En los claros, todas las variables fueron significativamente mayores en la ES, excepto la DRS ( $\mathrm{P}>0.05$, letras $\mathrm{a}, \mathrm{b})$; mientras que en BNP, los \% TDIR y \% TDIF, así como la DRS no difirieron significativamente $(\mathrm{P}>0.05)$. Destaca por magnitud la FRS, la cual fue casi tres veces mayor durante la ES, tanto para claros como BNP.

El ILuz (Fig. 1), fue significativamente más alto para los claros respecto a $\mathrm{BNP}$, independientemente de la estación $\left(\mathrm{P}<0.01^{* *}\right)$. Por otra parte, el ILuz fue significativamente más alto en la ES respecto de la ELL para los claros $(\mathrm{P}<0.05)$; mientras que no hubo diferencias estacionales significativas para el BNP $(\mathrm{P}>0.05)$.

Tamaño, zona y antigüedad de los claros: Para todas las variables, no hubo una interacción significativa entre tamaño y zona dentro del claro $(P>0.20)$, ni entre centro y borde de los claros, independientemente del tamaño $(\mathrm{P}>0.10)$. Tampoco hubo interacción
$(P \geq 0.07)$ entre tamaño y antigüedad. En los claros grandes, no hubo diferencias $(\mathrm{P}>0.05)$ en ninguna de las variables. Con respecto a los claros medianos, sólo la FRS fue no significativa, $(\mathrm{P}=0.15)$, mientras que para los pequeños todas las variables fueron diferentes $(\mathrm{P} \leq 0.02)$ excepto el \% DA $(\mathrm{P}=0.80)$ y el \% TDIF $(0.89)$. En los claros antiguos, los grandes difirieron de los medianos y pequeños, y presentaron mayores valores para todas las variables a excepción del IAF (Cuadro 2, letras a,b), los claros medianos y pequeños no difirieron significativamente excepto para \% TDIF. De los claros recientes, sólo los grandes mostraron diferencias $(\mathrm{P}<$ 0.05 , letras a,b) para \% DA y \% TDIF, siendo sus valores superiores respecto a los medianos y pequeños (Cuadro 2).

El ILuz fue significativamente mayor en claros recientes que en antiguos sólo en el caso de los medianos $(\mathrm{P}<0.01)$. Los claros recientes no mostraron diferencias entre tamaños $(\mathrm{P}>0.05)$; mientras que en los antiguos, el ILuz fue mayor en claros grandes respecto de los medianos y pequeños (Fig. 2a). No se encontró diferencias significativas del ILUz respecto a la posición dentro de claros (centro y borde); sin embargo, los claros grandes mostraron mayor Iluz tanto en el centro como

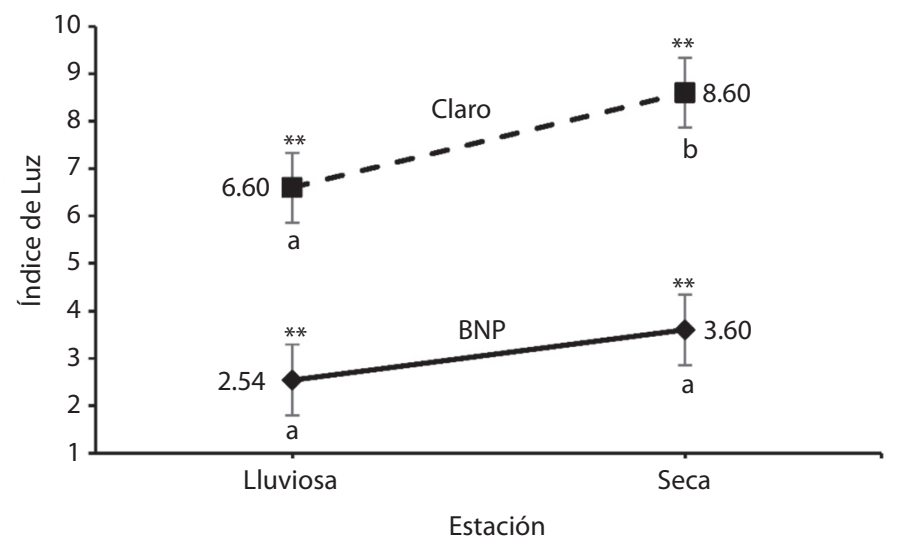

Fig. 1. Índice de luz para claros y BNP en las estaciones seca y lluviosa. Barras verticales indican error estándar, asteriscos diferencias estadísticamente significativas entre claros y BNP en cada estación $(\mathrm{P}<0.01)$. Letras diferentes indican diferencias estadísticamente significativas entre estaciones $(\mathrm{P}<0.05)$.

Fig. 1. Light index for gaps and BNP in the dry and rainy seasons. Vertical bars indicate standard errors; asterisks are statistically significant differences between gaps and BNP for a given season $(\mathrm{P}<0.01)$. Different letters indicate statistically significant differences between seasons $(\mathrm{P}<0.05)$. 
CUADRO 2

Estimados de las variables de la estructura del dosel y de luz para claros de diferente tamaño y antigüedad

TABLE 2

Estimates for canopy structure and light variables for gaps of different size and age

\begin{tabular}{|c|c|c|c|c|}
\hline & ıaño/edad & Antiguo & Reciente & p-valor \\
\hline \multirow[t]{3}{*}{ IAF } & Grande & $3.8(0.1)^{\mathrm{b}}$ & $3.9(0.1)^{\mathrm{a}}$ & 0.53 \\
\hline & Mediano & $4.9(0.1)^{\mathrm{a}}$ & $4.0(0.1)^{\mathrm{a}}$ & $<0.01$ \\
\hline & Pequeño & $4.8(0.3)^{\mathrm{a}}$ & $4.0(0.2)^{\mathrm{a}}$ & 0.02 \\
\hline \multirow[t]{3}{*}{$\% \mathrm{DA}$} & Grande & $5.0(0.3)^{\mathrm{a}}$ & $6.0(0.4)^{\mathrm{a}}$ & 0.08 \\
\hline & Mediano & $2.6(0.1)^{\mathrm{b}}$ & $4.5(0.3)^{\mathrm{b}}$ & $<0.01$ \\
\hline & Pequeño & $3.9(0.5)^{b}$ & $3.7(0.6)^{b}$ & 0.80 \\
\hline \multirow[t]{3}{*}{$\%$ TDIR } & Grande & $10.2(0.8)^{\mathrm{a}}$ & $11.5(1.1)^{\mathrm{a}}$ & 0.32 \\
\hline & Mediano & $5.0(0.3)^{\mathrm{b}}$ & $10.2(1.1)^{\mathrm{a}}$ & $<0.01$ \\
\hline & Pequeño & $3.6(0.5)^{\mathrm{b}}$ & $12.9(0.8)^{\mathrm{a}}$ & $<0.01$ \\
\hline \multirow[t]{3}{*}{$\%$ TDIF } & Grande & $7.9(0.5)^{\mathrm{a}}$ & $9.6(0.7)^{\mathrm{a}}$ & 0.05 \\
\hline & Mediano & $4.2(0.2)^{b}$ & $7.2(0.5)^{b}$ & $<0.01$ \\
\hline & Pequeño & $6.3(0.8)^{\mathrm{ab}}$ & $6.1(1.0)^{b}$ & 0.89 \\
\hline \multirow[t]{3}{*}{ DRS } & Grande & $46.6(4.1)^{\mathrm{a}}$ & $39.8(3.8)^{\mathrm{a}}$ & 0.22 \\
\hline & Mediano & $20.1(1.5)^{b}$ & $34.8(3.6)^{\mathrm{a}}$ & $<0.01$ \\
\hline & Pequeño & $22.0(3.3)^{\mathrm{b}}$ & $42.7(6.1)^{\mathrm{a}}$ & $<0.01$ \\
\hline \multirow[t]{3}{*}{ FRS } & Grande & $17.2(1.5)^{\mathrm{a}}$ & $17.9(2.2)^{\mathrm{a}}$ & 0.78 \\
\hline & Mediano & $11.1(1.0)^{\mathrm{b}}$ & $14.4(2.1)^{\mathrm{a}}$ & 0.15 \\
\hline & Pequeño & $9.0(1.1)^{\mathrm{b}}$ & $16.5(3.6)^{\mathrm{a}}$ & $<0.01$ \\
\hline
\end{tabular}

IAF: índice de área foliar; \% DA: porcentaje de dosel abierto; \% TDIR: porcentaje transmitido de luz directa; \% TDIF: porcentaje de luz difusa; DRS: duración promedio en minutos de los rayos de sol; FRS: frecuencia o número de rayos de sol de una duración específica. Números en paréntesis son errores estándar.

Letras diferentes indican diferencias estadísticamente significativas para la misma variable entre tamaños de claro $(\mathrm{P}<0.05)$. P-valor corresponde al nivel de significación para las diferencias en la antigüedad de los claros.

Different letters indicate statistically significant differences for a variable among gap sizes $(\mathrm{P}<0.05)$. The $\mathrm{P}$-value is the significance level for differences in gap age. Numbers in parenthesis are standard errors.

en el borde, que los claros medianos y pequeños $(\mathrm{P}<0.05)$ (Fig. 2b).

\section{DISCUSIÓN}

El IAF promedio coincide con lo observado para otros bosques tropicales (Meir, Grace \& Miranda, 2000; Asner, Scurlock \& Hicke, 2003; Negrón, Ribeiro, Silva, Goulden \& Miller, 2009). El bajo \% DA (máximo $<15 \%$ ) concuerda con estudios en bosques tropicales y templados (Ostertarg, 1998; Figueroa \& Lusk, 2001; Acevedo et al., 2003; Poorter \& Arets, 2003; Lusk, Chazdon, \&
Hofmann, 2006; Montgomery \& Chazdon, 2002; Lusk et al., 2006).

Las variables estructurales del dosel (IAF y $\%$ DA) y las estimaciones relativas al régimen lumínico (excepto FRS, en ELL) difirieron claramente entre BNP y claros. Exceptuando el IAF, las variables estudiadas prácticamente duplican su valor en los claros, lo que podría tener impacto sobre los procesos de regeneración en el sotobosque.

Las diferencias estacionales encontradas podrían relacionarse con diferencias en la sensibilidad de las especies a la sequía (ángulo foliar y deciduidad) resultado de la duración del período de déficit hídrico en el suelo 

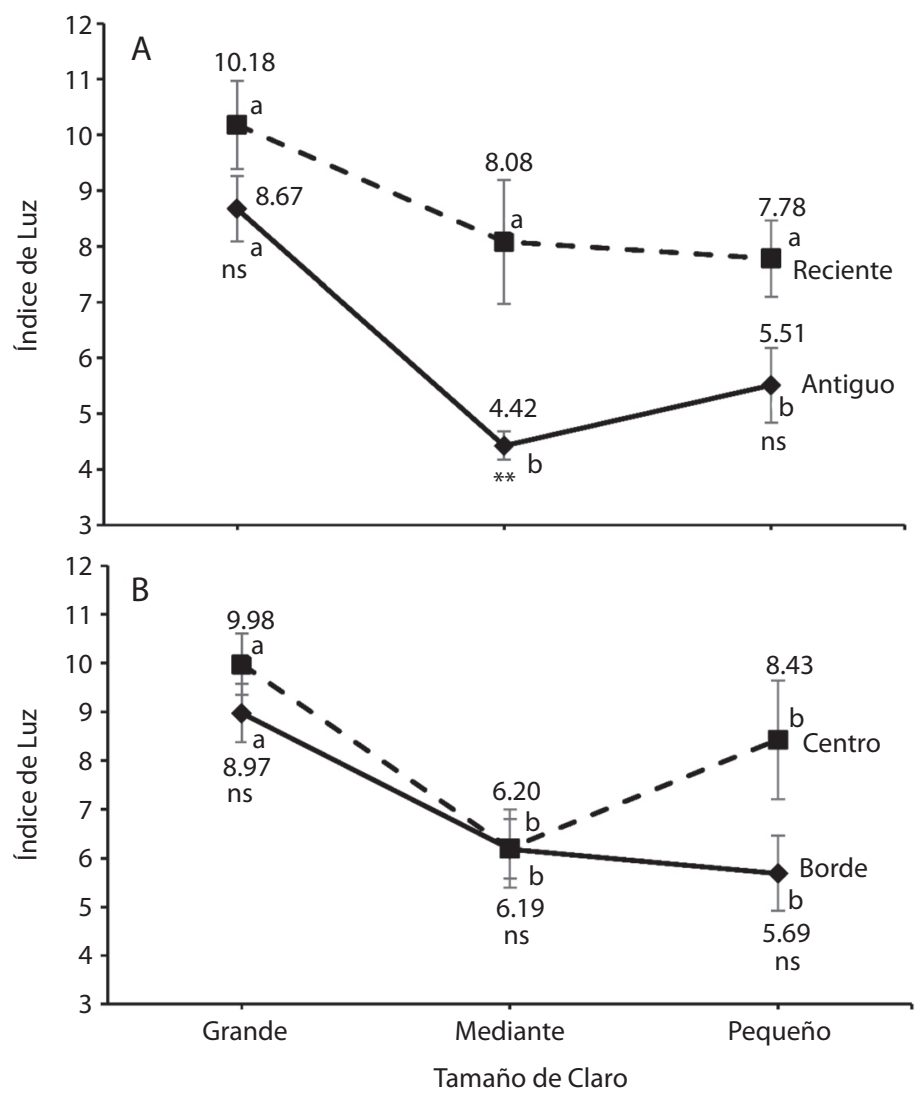

Fig. 2. Índice de luz para tamaño de claros según: A. antigüedad; B. posición (centro-borde). Barras verticales indican error estándar, asteriscos diferencias estadísticamente significativas entre claros del mismo tamaño $(\mathrm{P}<0.01)$. Letras diferentes indican diferencias estadísticamente significativas entre tamaños de claros $(\mathrm{P}<0.05)$.

Fig. 2. Light index estimates for: A. gap age vs. gap size; B. relative position with respect to the gap center (center-border) vs. gap size. Vertical bars indicate standard errors, asterisks indicate statistically significant differences between A. recentold; B. center-border $(\mathrm{P}<0.01)$. Letters indicate statistically significant differences among gap sizes $(\mathrm{P}<0.05)$.

y la mayor demanda evaporativa durante la época seca. Aunque las diferencias de la estructura del dosel entre estaciones parece ser pequeña, nuestros resultados indican que su impacto sobre el régimen de luz en el sotobosque no es despreciable, especialmente en los claros, donde las diferencias estructurales producen diferencias estacionales en radiación directa y difusa, así como en la FRS. El Iluz fue significativamente más alto en ES tanto en claros como en BNP, indicando una mayor transmisión de luz al sotobosque. Una posible explicación es que la infiltración de rayos de sol, desde el dosel hasta el sotobosque, está influenciada por la estructura del dosel; la geometría de los claros (Turnbull \& Yates, 1993), y por variaciones en el ángulo solar y nubosidad (Endler, 1993). Los claros grandes, a diferencia de los medianos y pequeños, no difieren en ninguna variable respecto a su antigüedad. Una posible explicación es que el proceso de cierre de un claro grande es a muy largo plazo. En este sentido, los cambios estacionales en la estructura del dosel en los claros medianos modifican todas las variables consideradas, excepto la FRS. Por otra parte, en claros pequeños, si bien no se modifica la estructura del dosel (IAF y \% DA) como en los medianos, se alteran significativamente las variables relacionadas con la luz directa 
(\% TDIR, DRS y FRS). Este resultado destaca que una alteración no fácilmente detectable de la estructura del dosel, puede producir cambios significativos en la incidencia de luz directa en el sotobosque. En tal sentido, el ILUz es una variable apropiada para detectar con fidelidad las variaciones del ambiente de luz en claros de diferentes tamaños, destacándose la interacción antigüedad vs. tamaño del claro: el tamaño no tiene impacto en el Iluz en claros recientes, pero si en claros antiguos.

Nuestros resultados muestran que en $\mathrm{SN}$, la cantidad de luz que llega al sotobosque es baja, tanto en BNP como en claros. Sin embargo, existen pequeñas pero significativas diferencias en la disponibilidad de luz, tanto por efecto de la estacionalidad como por la magnitud de las perturbaciones. El conocimiento de los factores que condicionan la disponibilidad de luz en el sotobosque, donde se inicia la regeneración de los árboles, es crucial en $\mathrm{SN}$ debido a su condición de ecosistema con limitaciones energéticas y es fundamental para futuros planes de restauración y conservación de la diversidad e integridad de estos bosques.

\section{AGRADECIMIENTOS}

Al Consejo de Desarrollo Científico, Humanístico, Tecnológico y de las Artes (CDCHTA) de la Universidad de Los Andes por su financiamiento bajo el proyecto F0-703-1001-A. A Misión Ciencia-FONACIT, Venezuela, 200601148. A Ancelmo y Dani Dugarte por su cooperación en las actividades de campo.

\section{RESUMEN}

El ambiente lumínico en el sotobosque de las selvas nubladas es muy heterogéneo y está determinado por la composición de especies, la estructura del dosel, las condiciones de sitio y la estacionalidad. Este estudio se realizó en la selva nublada San Eusebio, Venezuela (2 300-2 500 m.s.n.m.). El impacto de las variaciones estructurales del dosel en la disponibilidad de luz del sotobosque fue estimado durante la estación seca (diciembre-febrero) y lluviosa (marzo-noviembre), tanto en claros de diferente tamaño como en bosque no perturbado (BNP). Se tomaron fotografías hemisféricas para estimar el porcentaje de dosel abierto, índice de área foliar, porcentajes de luz directa y luz difusa transmitida, duración y frecuencia de rayos de sol. Se calculó un índice de luz a partir de las proporciones relativas de luz directa y difusa transmitida al sotobosque. Para la mayoría de las variables, hubo diferencias significativas entre las estaciones, así como entre claros y BNP. El índice de luz fue bajo ( 0.25 a 26 de un máximo $=100)$, incluso para los claros más grandes, indicando un ambiente de luz muy oscuro en el sotobosque, particularmente, en BNP en la estación lluviosa. Patrones e interacciones entre los factores fueron analizados (BNP vs. claros, tamaño de claro, posición en el claro y estacionalidad) con un diseño ANOVA de efectos mixtos y medidas repetidas. Los resultados mostraron que la cantidad de luz que llega al sotobosque es baja, tanto en BNP como en claros. Sin embargo, existieron diferencias pequeñas pero significativas en la disponibilidad de luz, tanto por efecto de la estacionalidad como por la magnitud de las perturbaciones. Estas diferencias podrían contribuir a explicar la dinámica de la regeneración de especies en estos bosques. El conocimiento de los factores que condicionan la disponibilidad de luz en el sotobosque, donde se inicia la regeneración de los árboles, es crucial en selvas nubladas debido a las limitaciones energéticas de este ecosistema, y podría ser fundamental para futuros planes de restauración y conservación de la diversidad e integridad de estos bosques.

Palabras clave: claros, fotografías hemisféricas, índice de luz, rayos de sol, luz directa y difusa.

\section{REFERENCIAS}

Acevedo, M., Ataroff, M., Monteleone, S., \& Estrada, C. (2003). Heterogeneidad estructural y lumínica del sotobosque de una selva nublada andina de Venezuela. Interciencia, 28, 394-403.

Asner, G. P., Scurlock, J. O., \& Hicke, J. A. (2003). Global synthesis of leaf area index observations: implications for ecological and remote sensing studies. Global Ecology \& Biogeography, 12, 191-205.

Brokaw, N. (1985). Gap-Phase Regeneration in a Tropical Forest. Ecology, 66, 682-687.

Canham, C. D. (1988). An index for understory light levels in and around canopy gaps. Ecology, 69, 1634-1638.

Cescatti, A., \& Niinemets, U. (2004). Leaf to Landscape. In W. K. Smith, T. C. Vogelmann \& C. Chritchley (Eds.), Photosynthetic Adaptation: Chloroplast to Landscape (pp. 42-84). New York, USA: Springer.

Endler, J. A. (1993). The Color of Light in Forests and Its Implications. Ecological Monographs, 63, 1-27.

Figueroa, J. A., \& Lusk, C. H. (2001). Germination requirements and seedling shade tolerance are not correlated in a Chilean temperate rain forest. New Phytologist, $152,483-489$. 
Frazer, G. W., Canham, C. D., \& Lertzman, K. P. (1999). Gap Light Analyzer (GLA), Version 2.0: Imaging software to extract canopy structure and gap light transmission indices from true-color fisheye photographs. New York, USA: Simon Fraser University, Burnaby, BC, and the Institute of Ecosystem Studies, Millbrook. Available in http://www.rem.sfu.ca/forestry/ index.htm or http://www.ecostudies.org

García, C., Azócar, A., \& Rada, F. (1995). Photosynthetic acclimation to light in juveniles of two cloud forest tree species. Tree, 10, 114-124.

Hogan, K. P., \& Machado, J. L. (2002). La luz solar: consecuencias biológicas y su medición. In M. R Guariguata \& G. H. Kattan (Eds.), Ecología y Conservación de Bosques Neotropicales (pp. 119-143). Cartago, Costa Rica: LUR.

Luna, I., Velázquez, A., \& Velázquez, E. (2001). México. In M. Kapelle \& A. D. Brown (Eds.), Bosques Nublados del Neotrópico (pp. 183-229). Costa Rica: INBio.

Lusk, C. H., Chazdon, R., \& Hofmann, G. (2006). A bounded null model explains juvenile tree community structure along light availability gradients in a temperate rain forest. Oikos, 112, 131-137.

Márquez, O. (1990). Génesis de una secuencia de suelos en el Bosque Experimental San Eusebio, La Carbonera. Estado Mérida. Revista Forestal Venezolana, $32,133-150$

Meir, P., Grace, J., \& Miranda, A. (2000). Photographic method to measure the vertical distribution of leaf area density in forests. Agricultural and Forest Meteorology, 102, 111-105.

Montgomery, R. A., \& Chazdon, R. L. (2002). Light gradient partitioning by tropical tree seedlings in the absence of canopy gaps. Oecologia, 131, 165-174.

Negrón, R., Ribeiro, H., Silva, A., Goulden, M. L., \& Miller, S. (2009). An improved estimate of leaf area index based on the histogram analysis of hemispherical photographs. Agricultural and Forest Meteorology, 149, 920-928.

Nobis, M., \& Hunziker, U. (2005). Automatic thresholding for hemispherical canopy-photographs based on edge detection. Agricultural and Forest Meteorology, $128,243-250$.

Ostertarg, R. (1998). Belowground effects of canopy gaps in a tropical wet forest. Ecology, 79, 1294-1304.

Pearcy, R. W. (2007). Responses of Plants to Heterogeneous Light Environments. In F. Pugnaire, \& F. Valladares (Eds.), Functional Plant Ecology (pp. 213255). New York, USA: Marcel Dekker Inc.

Poorter, L., \& Arets, E. J. (2003). Light environment and tree strategies in a Bolivian tropical moist forest: an evaluation of the light partitioning hypothesis. Plant Ecology, 166, 295-306.

Ramos, M. C., \& Plonczak, M. A. (2007). Dinámica sucesional del componente arbóreo luego de un estudio destructivo de biomasa en el Bosque Universitario San Eusebio, Mérida Venezuela. Revista Forestal Venezolana, 51, 35-46.

SAS. (2004). SAS/STAT 9.1. SAS, NC. USA: Institute Inc.Cary.

Schwarzkopf, T., Riha, S. J., Fahey, T. J., \& DeGloria, S. (2011). Are could forest tree structure and environment related in the Venezuela Andes? Austral Ecology, 36, 280-289.

Turnbull, M. H., \& Yates, D. J. (1993). Seasonal Variation in the Red/Far-Red Ratio and Photon Flux Density in an Australian Sub-Tropical Rainforest. Agricultural and Forest Meteorology, 64, 111-127.

Zhang, Y. C., Jing, M., \& Miller, J. R. (2005). Determining digital hemispherical photograph exposure for leaf area index estimation. Agricultural and Forest Meteorology, 133, 166-181. 
